\title{
DIE ERFASSUNG \\ DES WASSER- UND WÄRMEHAUSHALTS \\ DER ATMOSPHÄRE
}

\section{Paul Schaufelberger}

\section{PROBLEMSTE LLUNG}

Der Kreislauf des Wassers in der Atmosphäre ist bekannt: Das Wasser verdunstet über offener Wasserfläche, also in der Hauptsache von Meeren, fällt als Niederschlag teilweise auf die Kontinente und kehrt durch die Flüsse zum Meer zurück. Auf dem Festland beobachten wir weiter: Niederschlag, Oberflächenabfluß, Versickerung und Verdunstung. Dazu schreibt K. Keilhack (7): «Das Wasser welches als Regen, Hagel, Schnee, Reif und Tau auf die Erde niederfällt, hat ein dreifaches Schicksal: a) ein Teil der Niederschläge geht durch die Verdunstung sogleich wieder in die atmosphärische Luft zurück; b) ein zweiter Teil wird auf der Erdoberfläche sogleich den offenen Wasserläufen zugeführt und von diesen weiter befördert; c) ein dritter Teil endlich tritt in den Boden ein. - Eine Lehrmeinung, die so alt wie falsch und bis heute noch nicht ganz ausgerottet ist, sagt, daß von dem Niederschlagswasser je ein Drittel von diesen drei Vorgängen betroffen wird.»

O. Schoenefeldt (25) faßt die Erfahrungen der Hydrologie hinsichtlich des Wasserhaushaltes dahin zusammen: «Für die Betrachtung des Wasserhaushaltes im langjährigen Durchschnitt gilt für das Festland:

\section{Landniederschlag $(N)=$ Landabfluß $(A)+\operatorname{Landverdunstung~}(V) . »$}

Der Landabfluß umfaßt das Oberflächenwasser, das die Flüsse direkt speist, und das Sickerwasser, das im Boden versickert und auf unterirdische Wege ins Fluß-System oder Meer gelangt. Er führt das Wasser langsamer dem Flusse zu, weshalb die Tropenflüsse während der Trockenzeit nicht versiegen. Das zeigt sehr klar die Hydrologie der Meseta Central von Costa Rica.

Hydrologie der Meseta Central von Costa Rica

\begin{tabular}{|c|c|c|c|c|c|c|c|c|c|c|c|c|}
\hline Niederschlag m & $\mathrm{J}$ & $\mathrm{F}$ & M & A & M & $\mathrm{J}$ & $\mathrm{J}$ & A & S & $\mathrm{O}$ & $\mathrm{N}$ & $\mathrm{D}$ \\
\hline $\begin{array}{l}\text { San José (16) } \\
\text { Abflu } \beta 1 / \text { sek. }\end{array}$ & $15)^{5,4}$ & 2,8 & 9,7 & 46,7 & 221,8 & 258,3 & 207,5 & 244,2 & 348,9 & 342,4 & 150,6 & \\
\hline Rio Torres & 1350 & 1200 & 1350 & 1300 & 1380 & 1450 & 1440 & 1400 & 2280 & 3250 & 2850 & \\
\hline Río Titiribi & 2180 & & 1850 & 186 & 0 & & & & & 300 & $3 r_{4} O$ & \\
\hline \multicolumn{13}{|l|}{ Rio María } \\
\hline & 850 & 680 & 750 & & & & & & & & 3850 & \\
\hline io & 2930 & 4350 & 4700 & 485 & 5350 & & 69 & 5600 & & 9300 & 8360 & \\
\hline ío $s$ & 1120 & 980 & 1050 & 1300 & 1450 & 1850 & 1640 & 1980 & 2230 & 3830 & 2830 & \\
\hline
\end{tabular}

Das Maximum des Abflusses fällt in das Ende der Regenzeit, das Minimum, mit Ausnahme des Río Virillas, gegen das Ende der Trockenzeit. Boden und Untergrund speichern also einen Teil des Wassers der Regenzeit und geben es während der Trokkenzeit an die Flüsse $a b$, so daß sie nicht austrocknen und während des ganzen Jahres Wasser führen.

Von den drei Größen lassen sich Niederschlag und Abfluß bestimmen, aber Niederschlag - Abflu $\beta=$ Verdunstung entspricht nicht den mit der Wild'schen Waage meßbaren Verdunstung von freier Wasseroberfläche.

\section{WASSERVERDUNSTUNG}

Über die Wasserverdunstung des Kreislaufes des Wassers berichtet DrenkHahN (4) : «Das Wasser verdunstet bei jeder Temperatur, auch im festen Zustande als Eis 
oder Schnee. Mit zunehmender Temperatur steigt jedoch die Verdunstung erheblich, die Temperaturverhältnisse sind als ausschlaggebender Faktor für die Verdunstung anzusehen. Außerdem wird die Verdunstungsmenge beeinflußt von der Größe der Luftbewegung, insofern, als durch diese die Ausbildung einer wasserdampfgesättigten untern Luftschicht vermindert wird und immer neue aufnahmsfähige Luftmassen zugeführt werden, ferner von der relativen Feuchtigkeit der Luft und schließlich vom Luftdruck, dem die Verdampfungsgeschwindigkeit umgekehrt proportional ist. Bei der Verdunstung des Wassers vom Erdboden, der sogenannten ,Landverdunstung', kommen als weitere Einflüsse hinzu die Neigungsverhältnisse, Bodendeckung (Vegetation), Material, Größe und Lagerung der Bodenteilchen, 'Tiefenlage des Grundwasserspiegels und Häufigkeit der Benetzung der Erdoberfläche. Wegen dieser mannigfaltigen Einflüsse ist die Anstellung zuverläßiger Verdunstungsmessungen von freien Wasserflächen und besonders von Landflächen mit großen Schwierigkeiten verbunden, und die aus den Messungen erhaltenen Werte sind mit großer Vorsicht aufzunehmen. - Es ist seither nicht gelungen, über die Größe der Landverdunstung zuverläßige Werte durch Messung zu erlangen, da bei allen Meßverfahren Eingriffe in die natürlichen Verhältnisse vorgenommen und wichtige Vorgänge ausgeschaltet werden, die sich in der Natur während der Verdunstung abspielen.»

Nun unterscheidet man zwischen unproduktiver oder direkten und produktiver Verdunstung, worunter die indirekte durch die Transpiration der Pflanzen verstanden wird. Über diese finden wir in der Literatur zahlreiche Angaben. So berichtet der Höhlenforscher W. von KNeBEL (27): «Der Verbrauch einer Buche von mittlerer Größe ( 50 bis 60 Jahre alt) erreicht während der Vegetationsperiode durchschnittlich $10 \mathrm{~kg}$ pro Tag. Da auf einem Hektar etwa 1300 Bäume dieser Größe sich befinden, so verliert ein Wald von dieser Fläche während der gesamten Vegetationsperiode des Jahres $2300000 \mathrm{~kg}$ Wasser, d. i. $2300 \mathrm{~m}^{3} . »$

Fitting, Jost, Schenk, Karsten (6) machen folgende Angaben über die Transpiration: «Die Leistungen der Blätter im Verdunsten von Wasser sind denn auch geradezu überraschend. Eine kräftige Pflanze, beispielsweise eine Sonnenblume von Mannshöhe verdunstet an einem hellen Tage über einen Liter Wasser, und man hat festgestellt, daß ein Morgen mit Kohlpflanzen in vier Monaten zwei Millionen Liter, ein solcher mit Hopfen drei bis vier Millionen Liter Wasser verdunstet. Für eine einzeln stehende Birke mit etwa 200000 Blättern berechnete von HoEnel, das verdunstete Wasser an einem heißen, trockenen Tage auf ca. 300 bis 400 Liter, im Tagesdurchschnitt auf 60 bis 70 Liter. Ein Hektar Buchenhochwald verdunstet im Durchschnitt 20000 Liter täglich. Auf je $100 \mathrm{~g}$ Blattsubstanz berechnet, verbraucht in einer Vegetationsperiode die Rotbuche 75 Liter, die Tanne aber nur 7 Liter. Im Durchschnitt verdunstet eine Pflanze für jedes Gramm Trockensubstanz, das sie in ihren oberirdischen Teilen bildet, 250 bis $900 \mathrm{~g}$ Wasser.» P. VAgeler (26) macht Angahen über den Wasserverbrauch tropischer und subtropischer Kulturpflanzen: «Man wird im großen und ganzen annehmen können, daß zur Produktion einer Tonne trockener, organischer Substanz rund 400 Tonnen Wasser nötig sind. Je nach der Massenproduktion der natürlichen Vegetation schwankt die Entziehung von Bodenwasser, das demnach nur vorübergehend als Bodenfeuchtigkeit in genetischem Sinne wirkt, je Hektar zwischen Null in der Wüste und etwa 6000 Tonnen im Urwald durch alle Zwischenwerte. Eine Tonne Wasser je Hektar entspricht einem Zehntelmillimeter Niederschlag, wonach auszurechnen ist, daß allein auf diese Weise von den Niederschlägen ein sehr erheblicher Bruchteil namentlich in den an Vegetation reichen Gegenden verloren geht, der der Bodenfeuchtigkeit nur sehr bedingt und vorübergehend zuzurechnen ist. Trägt man dem allen Rechnung, so ergibt sich bei den üblichen Pflanzzeiten für Maximalernten liefernde Bestände der einzelnen wichtigsten Kulturpflanzen ausgedrückt in $\mathrm{mm}$ Regenhöhe etwa folgender Wasserbedarf je Hektar: 
Getreidearten

Mais und Hirse

Zuckerrohr

$120-150$

200-250

$400-500$

Knollen- und Wurzelfrüchte 300--400

Oelfrüchte

$120-150$

Hanf und Jute

200-250

Baumwolle

Kokospalmen
Oelpalmen

Agaven

Kaffee

Kakao

Tee

Wein

Obstbäume
$600-700$

200-250

250-300

$350-400$

$100-150$

$100-150$

$100-200$

Das sind alles scheinbar sehr niedrige Bedarfsziffern. Aber wie oft trügt der Schein auch hier. Sie sind tatsächlich sehr hoch, denn mit Rücksicht auf die Verdunstungsverluste der Niederschläge an den Pflanzen, aus dem Boden und durch die Versickerungsverluste, die in diesen Zahlen nicht enthalten sind, sind alle im großen Durchschnitt mit 5 zu multiplizieren, da kaum jemals mehr als $20 \%$ der Regen den Pflanzen wirklich zugute kommt.»

Nach diesen Ausführungen ergibt sich für die Kaffeezone von Chinchina, Kolumbien, für das Jahr folgende Wasserbilanz, nach den vorliegenden meteorologischen Angaben (5):

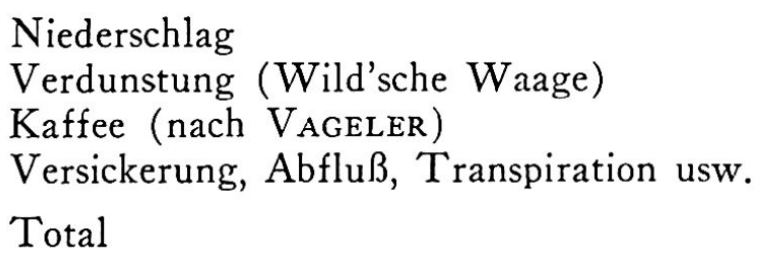

\begin{tabular}{cc}
$\mathrm{mm}$ & $\mathrm{mm}$ \\
2857,3 & \\
& 533,0 \\
& 300 \\
& 2024,3 \\
\hline 2857,3 & 2857,3
\end{tabular}

Über den Wasserhaushalt deutscher Böden schreibt W. LAatsch (8) : «Für lange Zeiträume gilt demnach die Gleichung: Niederschlag $=$ unproduktive + produktive Verdunstung + Sickerwasser. Abgesehen wird hier von den Verhältnissen steiler Hänge an denen ein erheblicher Teil des Niederschlages oberflächlich abfließt. Übersicht man den Verbleib der monatlichen Niederschlagsmengen, so darf die Speicherungsfähigkeit, d. h. die wasserhaltende Kraft des Bodens, nicht vernachläßigt werden. Wenn der Niederschlag eines Monats die Summe aus Verdunstung und Sickerwasser in dieser Zeit übersteigt, ist ein Teil des Niederschlags im Boden, d. h. in der oberen, durchwurzelten Zone der Erdrinde aufgespeichert worden. Umgekehrt kann die Summe aus Verdunstung und Sickerwasser größer als der Niederschlag in der Zeiteinheit sein. In diesem Falle muß ein Teil des zu Beginn der Messung im Boden befindlichen Wassers durch Verdunstung oder Versickerung entwichen sein. Mithin gilt für grundwasserfreie Böden die Beziehung:

Niederschlag $=$ Verdunstung + Sickerwasser $+($ Speicherung - Aufbrauch $)$. Diese Gleichung ist in Anlehnung an die Wasserhaushaltformel der Wassertechniker: Niederschlag $=$ Abflu $\beta+$ Verdunstung + (Rücklage-Aufbrauch $)$ aufgestellt. Die letzte Formel bezieht sich jedoch nicht nur auf den an dieser Stelle allein interessierenden Boden - also die obere 1-4 m mächtige, durchwurzelte Lockerzone - sondern auf den ganzen vom atmosphärischen Wasser durchsickerten Teil des Erdinnern mit seinen sämtlichen Grundwasserstockwerken.»

H. LECHNER (11) hat die Wasserbilanz für das deutsche Bundesgebiet des Jahres 1951 studiert und macht folgende Feststellungen:

«Als Wasservorrat können die Niederschläge gelten, über die wir dank systematischer Wetterbeobachtungen ein hinreichend genaues Bild besitzen. Die Schwankungen in den Lagen geben im Mittel des Bundesgebietes eine Regendecke von $780 \mathrm{~mm}$. Bei $240000 \mathrm{~km}^{2}$ Bundesgebietsfläche entspricht dies einem Wasserschatz von 190 Mrd $\mathrm{m}^{3}$ im Durchschnitt der Jahre. Dazu müßte man noch die Wassermenge berücksichtigen, die von einigen Flüssen aus Niederschlagsgebieten außerhalb des Bundes- 
gebietes zugeführt wird. Dem Wasservorrat steht der Wasserbedarf gegenüber, der z. B. 1951 beim Trinkwasser 2,2 $\mathrm{Mrd} \mathrm{m}^{3}$ und beim Industrieverbrauch 4,4 $\mathrm{Mrd} \mathrm{m}^{3}$ ausmachte. Auch der Wasserbedarf der Land- und Forstwirtschaft läßt sich größenordnungsgemäß ermitteln. So sei ziemlich genau bekannt, daß im Wirtschaftsjahr erzeugten pflanzlichen Produkte 117 Mio t wogen. Man wisse auch ungefähr, daß zu $1 \mathrm{t}$ Pflanzentrockenmasse $400-500 \mathrm{~m}^{3}$ Wasser nötig sind. Hieraus ergebe sich ein annähernder Wasserbedarf der Landwirtschaft von rund $50 \mathrm{mrd} \mathrm{m}^{3}$. Der forstliche Wasserbedarf, in ähnlicherweise ermittelt .. 4,2 $\mathrm{Mrd} \mathrm{m}^{3} . \gg$

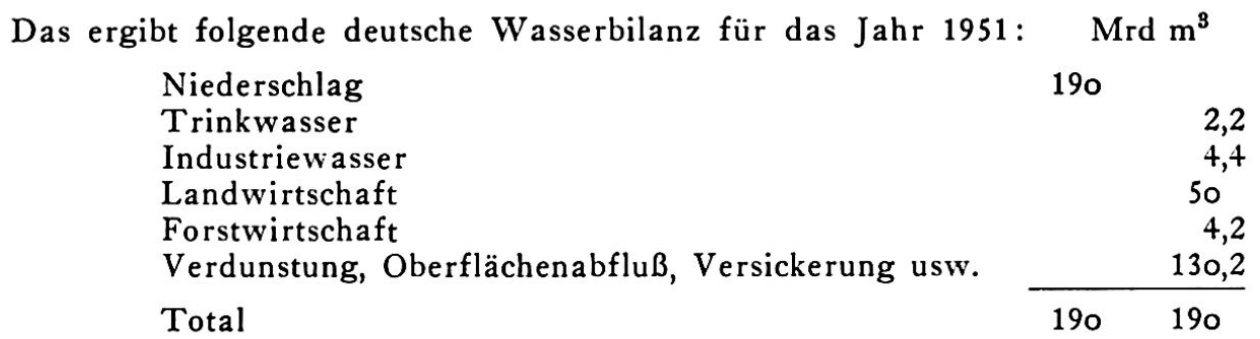

\section{LYSIMETER}

Lysimeter sind, nach E. Deichmann (2), «würfelförmige oder zylindrische in die Erde eingebaute, nach oben offene, mit Erde gefüllte Betonkasten von ca. $1 \mathrm{~m}$ Tiefe, von derem schrägen Boden das durchgesickerte Wasser abläuft zwecks Studium der Auswaschungsvorgänge gesammelt, gemessen und untersucht wird». Man kann also damit das tatsächliche Sickerwasser bestimmen und erhält die Gleichung:

Niederschlag - Sickerwasser - Verdunstung

$\mathrm{Da} \beta$ damit die «Verdunstung» bestimmt wird, geht aus den Ausführungen von B. RAMSAUER

(14) hervor:

«Es gibt einige Verfahren, die für größere Einzugsgebiete aus gegebenen meteorologischen Daten die Verdunstungshöhe mit ziemlicher Genauigkeit errechnen lassen. - Diese Tatsache enthebt uns jedoch keineswegs der Notwendigkeit, sowohl für größere Einzugsgebiete wie insbesondere für die Ermittlung des Mikroklimas, die Verdunstung täglich nach Höhe und Verlauf festzustellen. Unentbehrlich sind solche Beobachtungen für das Dränungsversuchswesen, da ohne Kenntnis der Verdunstung der Wasserhaushalt des Versuchsgebietes nicht ermittelt werden kann. Unbestritten ist dieses Ziel am sichersten mit Lysimetern zu erreichen, weshalb zu jedem kulturtechnischen Versuchsfeld eine solche Anlage gehört.»

Auf dieser Annahme beruht auch der Vorschlag von W. LaAtsch (8) zur Bestimmung der Durchfeuchtung deutscher Sandböden:

«Aus diesem Grunde muß bei der Berechnung der Verdunstung auf die Lysimeterbeobachtungen zurückgegriffen werden. Besäßen wir bereits ein langjähriges Mittel der Eberswalder Verdunstungsmessungen, so könnten wir für das Eberswalder Klima und die Bodenverhältnisse: Sandboden mit Grasnarbe und tiefstehendem Grundwasser - die Beziehungen zwischen der vom Sättigungsdefizit ausgehenden Verdunstungskraft und der wirklichen Verdunstung nach folgender Formel errechnen:

$$
V=D . a
$$

$V=$ Lysimeterverdunstung, $D=$ gemessenes Sättigungsdefizit der Luft, $a=$ errechneter Multiplikator.»

Über die tatsächlichen Resultate langjähriger Lysimeterbeobachtungen berichten C. PFAFF und W. Friedrich (13):

«Bei der Auswertung der Ergebnisse langjähriger Lysimeterversuche, die auf der Landw. Versuchsstation Limburgerhof durchgeführt wurden, machten die Verff. folgende Feststellungen bezüglich des Wasserhaushaltes des Bodens: Die durchschnittliche jährliche Niederschlagsmenge betrug $580 \mathrm{~mm}$. Im Sommer wurden künstliche Wassergaben $(50-100 \mathrm{~mm}$ ) gegeben, so weit dies zur Förderung des Pflanzenwachstums unerläßlich war. Dieser Nachteil mußte in Kauf genommen werden. Auf der andern Seite haben die Messungen im Limburgerhof den Vorteil, da $B$ sie in einer sonst selten erreichten Versuchsdauer und unter sorgfältig eingehaltenen Versuchsbedingungen mit einer großen Zahl von Vergleichsparzellen gewonnen wurden. Die Ergebnisse sind bei fehlendem Oberflächenabflu $\beta$ gekennzeichnet durch beträchtliche Versickerung in geschüttetem Boden meist $30 \%$ der Niederschläge und mehr.»

Darnach wäre die berechnete «Verdunstung» maximal rd. $70 \%$ oder $387 \mathrm{~mm} / \mathrm{Jahr}$.

Mit dieser Frage der Deutung der Lysimeterversuche befa $B t$ sich auch M. H. OlberTz (12), der andere Autoren zitiert: 
«Unter der Annahme, daß der Wasserhaushalt am Anfang und Ende eines Beobachtungsjahres etwa gleich ist, ein Oberflächenabfluß nicht eintritt und die Verdunstung auf Grund von Versuchen Wollny's, Buhler's und Hellriegel's mit $600 \mathrm{~mm}$ Jahr angenommen werden kann, ergibt sich folgende Wasserhaushaltsgleichung für die Untersuchung EBERMAYER's:

$$
\begin{aligned}
\text { Sickerwasser }+ \text { Verdunstung } & =\text { Niederschlag }+ \text { Kondensation } \\
991 \mathrm{~mm}+600 \mathrm{~mm} & =932 \mathrm{~mm}+x \\
x=656 \mathrm{~mm} & =70 \% \text { des Jahresniederschlages. }{ }^{\circ}
\end{aligned}
$$

Wieso die Verdunstung, die man nicht messen kann, in der Klimatologie und der Bodenkunde eine so große Rolle spielt, erfahren wir von E. BLANK (1) :

«Wie wir diesen Darstellungen (von PENCK) entnehmen können, sind es die Temperatur, die Niederschläge und die Verdunstung, welche ausschlaggebend für die Bodenentwicklung sind. Von E. Ramann sind sie infolgedessen auch als Großwerte des Klimas bezeichnet worden.»

$\mathrm{Da} \beta$ diese angeblichen Klimagroßwerte bei einer Nachprüfung völlig versagen, hat man vor allem in den Tropen festgestellt.

\section{VERDUNSTUNG ALS FUNKTION DES NIEDERSCHLAGS}

Die «Ernährung der Pflanze» (3) referiert über den Vorschlag von J. Mohr:

«Die Verdunstung des Bodens nahm mit dem Regenfall zu. Während bei einem Regenfall von $135 \mathrm{~mm}$ im September die Verdunstung im Evaporimeter $132 \mathrm{~mm}$ betrug, war sie im Boden nur $81 \mathrm{~mm}$. Im Monat Oktober mit $625 \mathrm{~mm}$ Regen betrug die Verdunstung im Verdunstungsmesser $151 \mathrm{~mm}$, vom Boden aber $139 \mathrm{~mm}$. MoHR betrachtet die Verdunstung als lineare Funktion des Regenfalls, also

$$
V=C+F \cdot R \text {, }
$$

worin $C$ ungefähr 60 und $F=1 / 8$ ist. Bei einem Regenfall von $68 \mathrm{~mm}$ im Monat würde also in diesem Klima die Verdunstung ungefähr ebenso groß sein wie der Regenfall: erst bei einem höheren Regenfall kann Wasser in den Boden eindringen. Im allgemeinen rechnet MонR, daß man sicherheitshalber die Grenze des Regenfalls, bei dem Wasser den Boden von oben nach unten durchsickern kann, auf 90-100 mm festzusetzen hat. Die Möglichkeit, daß der Boden den Bedingungen eines ariden Klimas unterliegt, hängt von der Länge der Zeit $a b$, in der der Regenfall zu gering ist. Es kommt aber auf darauf an, ob die vorhergehenden Monate mäßig feucht oder sehr feucht waren. Im ersten Fall zählt bereits der erste Monat mit geringem Regenfall als trocken; im zweiten Fall findet sich noch ein Wasservorrat im Boden, der die Trokkenzeit verkürzt.»

\section{DIE * BEFEUCHTUNG *}

Über die «Befeuchtung» schreibt P. VAGELER (26):

«Sowohl die Lang'schen wie die MEYER'schen Faktoren ergeben, namentlich wenn man sie auf die Maxima der klimatischen Faktoren, die dem Boden einzig ihren Stempel aufprägen, bezieht, für die Betrachtung sehr großer Gebiete beachtenswerte Fingerzeige. Aber der Ausnahmen sind zu viele, um die Regel zu bestätigen. Mit Recht weist aber JENNY darauf hin, daß man zwar Niederschlag und Verdunstung in irgend einer Form als ,Befeuchtung' zusammenfassen, aber in keinem Fall die davon gänzlich unabhängige Temperatur damit vereinigen dürfe. Er faßt die Böden in regionaler Betrachtung daher als Funktion zweier unabhängiger Variabeln, Befeuchtung und Temperatur, auf, nun bereits für minder große Gebiete, wie Nordamerika und Europa, mit unleugbarem Erfolge. Gegenüber den Übertreibungen der engen Gebundenheit von Boden und Klimafaktoren bleibt er sich dabei aber bewußt, daß eine nur klimatische Betrachtung nicht allen Böden gerecht zu werden vermag. Er stimmt vollständig mit der Idee überein, daß die Böden nur nach ihren Eigenschaften klassiert werden sollen, ohne dabei die großen genetischen Gesichtspunkte, die eine allgemeine Klassifizierung ermöglichen, zu vernachläßigen.»

W. LaAtsch (8) befaßt sich ebenfalls mit dem Regenfaktor und kommt zu folgendem Schluß: 
«Von bodenkundlicher Seite hat man bisher kaum versucht, den Durchfeuchtungswert N-V für verschiedene Gebiete Deutschlands oder Europas zu ermitteln. Wir verfügen lediglich über mehrere mathematische Beziehungen, die zwischen Niederschlag und Temperatur bzw. Luftfeuchtigkeit aufgestellt wurden, um dadurch Vergleichswerte für die Durchfeuchtung der Böden zu erhalten. - Richard Lang schlug als Vergleichswert den sogenannten

$$
\text { Regenfaktor }=\frac{\text { Niederschlag }}{\text { Temperatur }}
$$

vor. Diese Beziehung stimmt mit den natürlichen Verhältnissen jedoch keineswegs überein. Bei 0 Grad erreicht der Regenfaktor den Wert unendlich und je mehr die Temperatur ansteigt umso geringer müßten nach LANG die Unterschiede im Austrocknungszuwachs sein.»

Scheffer und Schachtschnabel (24) nehmen ebenfalls Stellung zum Regenfaktor:

«Nachdem man die klaren Zusammenhänge zwischen Bodenentwicklung und Klima erkannt hatte, suchte man diese durch zahlenmäßige Ausdrücke festzulegen. So führte LANG den Regenfaktor ein. Er ging dabei von der Erwägung aus, daß Niederschläge und Verdunstung die wichtigsten klimatischen Faktoren darstellen. Die Verdunstung wird ihrerseits hauptsächlich durch die Temperatur beeinflußt, während die Luftfeuchtigkeit und Windstärke (im Jahresmittel) für den gleichen Ort nur im engeren Grenzen schwanken. Da nach seinen Betrachtungen die Verdunstung annähernd proportional mit der 'Temperatur ansteigt, benutzt er zur Berechnung des Regenfaktors neben der Niederschlagsmenge $(\mathrm{N})$ die mittlere Temperatur ( $\mathrm{T}$ ).» Genau ins gleiche Horn stößt auch die Klimatologie, wie W. LAuer (10) beweist:

«Von der Grundtatsache ausgehend, daß bei steigender Temperatur die Verdunstung zunimmt, hat der Bodenkundler R. LANG den sog. , Regenfaktor' aufgestellt, indem er den Quotient zwischen Jahresniederschlag (in $\mathrm{mm}$ ) und Jahresmitteltemperatur (in ${ }^{\circ} \mathrm{C}$ ) bildet. Die Regenmenge wird also in diesem Faktor nach der Verdunstungsmöglichkeit bei der jeweiligen Temperatur korrigiert. Der Lang'sche Regenfaktor, der in der Hauptsache in der Bodenkunde praktische Verwendung fand, wurde von P. Hirth in seiner Isonotidenkarte für die ganze Erde dargestellt, die zugleich aber die Unbrauchbarkeit des Quotienten für die Klimatologie erwiesen hat. Der Temperatur ist in der LANG'schen Formel eine zu große Wirkung auf die Verdunstung beigemessen, mit anderen Worten bei geringem Anstieg der Temperatur wird eine zu große Erhöhung der Verdunstung angenommen. Im Jahre 1926 hat dann E. DE MARTONNE eine neue Formel aufgestellt, die eine Verbesserung von LANG darstellt, indem er den Einfluß der Temperatur herabminderte und das Rechnen mit negativen Temperaturen dadurch vermied, daß er im Nenner den Wert plus 10 anbrachte.»

\section{DER REGENFAKTOR LANGS}

R. LANG (9) stellt die Befeuchtung in einem Koordinatensystem dar, mit der Regenmenge als Abszisse und der Temperatur als Ordinate. Er definiert nun:

$$
\begin{array}{lc}
\text { I. Regenfaktor }=\frac{\text { Jahresniederschlag in } \mathrm{mm}}{\text { Jahresmitteltemperatur }{ }^{\circ} \mathrm{C}} \\
\begin{array}{lc}
\text { II. Grenze } & \text { bei Regenfaktor } \\
\text { arid/semiarid } & 40 \\
\text { semiarid/semihumid } & 60 \\
\text { semihumid/humid } & 100 \\
\text { humid/perhumid } & 160
\end{array}
\end{array}
$$


Hier handelt es sich um reine Mathematik, indem die «Befeuchtung》 rein mathematisch als Fläche dargestellt und durch die Regenfaktoren 40, 60, 100 und 160 unterteilt wird. Kennen wir den Jahresniederschlag und die mittlere Jahrestemperatur, so wird rein mathematisch seine Befeuchtung bestimmt.

Ist nun diese vorerst mathematisch definierte Befeuchtung auch ein physikalischer Begriff, so muß sich deren Maßeinheit ins absolute Maß-System der Physik einbauen lassen. Wie ist nun der Niederschlag rein physikalisch zu messen? Die Meteorologie mißt die Regenhöhe in $0,1 \mathrm{~cm}$. Geologie und Hydrologie rechnen mit dem Regenvolumen: Liter pro Quadratmeter oder im absoluten Maß-System mit $0,1 \mathrm{~cm}^{3} / \mathrm{cm}^{2}$. Dies entspricht nach den Gesetzen der Physik einer Regenmasse von $0,1 \mathrm{gr} / \mathrm{cm}^{2}$ und bei $\mathrm{T}^{\circ} \mathrm{C}$ einer Regenwärme von T. $0,1 \mathrm{cal} / \mathrm{cm}^{2}$. Nach den Gesetzen der Thermik ergeben sich folgende Beziehungen:

Nun definiert Lang:

$$
\begin{aligned}
\text { Jahresregenwärme } \mathrm{call} \mathrm{cm}^{2} & =\text { Jahresregenmasse } \mathrm{gr} . T \mathrm{~T} \mathrm{~cm}^{2} \\
\text { Jahresregenmasse } \mathrm{gr} / \mathrm{cm}^{2} & =\frac{\text { Jahresregenwärme } \mathrm{cal}}{\mathrm{cm}^{2} T^{\circ} \mathrm{C}}
\end{aligned}
$$

$$
\text { Regenfaktor }=\frac{\text { Jahresregenmasse } g r}{c m^{2} . T^{\circ} C}=\frac{\text { Jahresregenwärme cal }}{c m\left(T^{\circ} C\right)^{2}}
$$

und Grenze

$\operatorname{arid} /$ semiarid

semiarid/semihumid

semihumid/humid

humid/perhumid
Regenfaktor

40
60
100
160

$\frac{\text { Jahresregenmasse } g r}{\mathrm{~cm}^{2} \cdot 1^{\circ} \mathrm{C}}$

4
6
10
16

$\frac{\text { Jahresregenwärme cal }}{\mathrm{cm}^{2}\left(1^{\circ} C\right)^{2}}$
4
6
10
16

Nun nimmt LANG an, daß sich bei jeder der fünf durch die Regenfaktoren definierten Befeuchtungen ein anderer Klimabodentyp bilden soll. Daß dies tatsächlich der Fall ist, beweist die Chemie:

\section{Langs Hypothese}

Jahresregenwärme cal $\mathrm{cm}^{2}\left(1^{\circ} \mathrm{C}\right)^{2}$

unter 4

$$
4-6
$$

$6-10$

$10-16$

über 16
Klimasol

Kaktussol

Bambussol

Humussol

Waldsol

Urwaldsol
Chemische Tatsachen

$\begin{array}{ccr}\text { Basengehalt } & \text { Humusgehalt } & \text { Auswaschungsfaktor } \\ \text { vmal/100g } & \% \% & \\ <10 & \text { ca. } 1 & +s i-c \text {-alk } \\ <10 & \text { ca. } 2 & +s i+c-\text { alk } \\ <10 & 3-5 & +s i+c+\text { alk } \\ <10 & 5-10 & +s i-c+\text { alk } \\ <10 & 10-20 & +s i-c \text {-alk }\end{array}$

Diese Klimabodentypen sind nachgewiesen auf vulkanischen Aschen in Kolumbien (17); auf basischen (18), intermediären (19) und sauren Eruptivgesteine (20), sowie auf Löß in Eurasien (23). Die Bodenlehre formuliert daher die LANG'schen Gesetze wie folgt (22):

Erstes Gesetz: die Befeuchtungen arid, semiarid, semihumid, humid und perhumid sind durch die Regenfaktoren 40, 60, 100 und $160 \mathrm{zu}$ definieren.

Zweites Gesetz: Die Klimasols oder Klimabodentypen sind basenarme endoperkolative Waldböden, in denen der Regenfaktor den Humusgehalt und den Auswaschungsfaktor bestimmt.

Wer nun beweisen will, daß die Regenfaktoren nicht stimmen, der muß den Nachweis erbringen, daß sich bei den durch die Regenfaktoren definierten «Befeuchtungsbereiche» basenarme Waldböden mit anderm Humusgehalt und anderen Auswaschungsfaktoren gebildet haben. 
LANG definiert die Befeuchtung mathematisch durch Jahresregenmasse in $\mathrm{gr} / \mathrm{cm}^{2}$ durch die mittlere Jahrestemperatur und nimmt an, daß sich bei jeder Befeuchtung, bei optimalen Bodenbildungsbedingungen, ein anderer Klimabodentyp bilde. Geologie, Petrographie, Chemie und Bodenlehre zeigen, daß diese Klimabodentypen sich tatsächlich bilden. Diese Tatsache läßt sich nicht wegdiskutieren.

\section{ZUSAMMENFASSUNG}

Man kennt den Kreislauf des Wassers in der Atmosphäre: Verdunstung, Niederschlag und Rückfluß zum Meer, aber alle Versuche diesen Kreislauf mathematisch zu definieren scheiterten an der Unmöglichkeit die Verdunstung zu messen.

LANG definiert die Befeuchtung mathematisch durch den Niederschlag und die Temperatur und nahm an, da $B$ sich bei jeder Befeuchtung ein anderer Klimabodentyp bilde, was sich durch die heute bekannten Tatsachen geologisch, petrographisch und chemisch beweisen läßt. Physikalisch zeigt der Regenfaktor die Gesetzmäßigkeiten zwischen Regenmasse, Temperatur und der Regenwärme und erfaßt somit den Wasser-und Wärmehaushalt des Kreislaufes des $W$ assers in der Natur. Die zur Verdunstung aufgewandte Energie führt der Regen der Erde wieder zu. Diese dem Boden jährlich zugeführte Energie bestimmt bei den endoperkolativen Waldböden das Endglied der Bodenentwicklung und diese steht im Gleichgewicht mit dem örtlichen Klima und der lokalen Waldvegetation.

\section{LITERATURANGABEN}

1. Blanck, E. (1949): Einführung in die genetische Bodenlehre. Göttingen. 2. Deichmann, E. (1949): ABC der Pflanzenernährung und Düngung. Limburgerhof. 3. - (1934): Die Ernährung der Pflanze. 3o/13-14. 4. Drenkhahn, L. (1927): Kreislauf des Wassers und Gewässerkunde. Berlin und Leipz!g. 5. - (1956): Federación Nacional de Cafeteros. Anuario Meteorológico. Chinchiná. 6. Firrunc, Jost, Schenk, Karsten (1917): Lehrbuch der Botanik für Hochschulen. Jena. 7. KeilHack, K. (1917: Grundwasser- und Quellenkunde. Berlin. 8. LaATSCH, W. (1938): Die Durchfeuchtungswerte der deutschen Sandböden. D. Ern. d. Pfl. 34/1. 9. LaNG, R. (1915): Versuch einer exakten Klassifikation der Böden in klimatischer und geologischer Hinsicht. Int. Mitt. f. Bodenkunde. 10. LAUER, W. (1952): Humide und aride Jahreszeiten in Afrika und Südamerika und ihre Beziehungen zu den Vegetationsgürteln. Bonner geogr. Abhdlg. H. 9. 11. Lechner, H. (1953): Wird unsere Wasserdecke zu knapp? Ref. in Kurz \& Bündig 7/2 (1954). 12. OlberTZ, H. M. (1957): Über die am Standort des Kulturbodens erfaßbaren Größen des Wasserhaushaltes. Berlin. 13. Pfaff, C. und Friedrich, W. (1954): Die Wasserbilanz des bewachsenen Bodens nach Lysimeterversuchen. Ref. in Kurz \& Bündig 7/20. 14. Ramsauer, B. (1938): Verdunstungsmesser.und Verdunstungsschreiber. D. Ern. d. Pfl. 34/2. 15. Schaufelberger, P. 1935: Un estudio geológico de la Meseta Central Occidental. Rev. d. Inst. d. Defensa de Café No 2. 16. - (1936): Algunos datos meteorológicos de Costa Rica. CNA, No 3-4. 17. - (1951): Los suelos de ceniza volcánica del Departamento de Caldas. Rev. Cafetera X/123. 18. - (1953): Tropische Verwitterung und Bodenbildung über Andesit und Diorit. Schweiz. Min. petr. Mitt. 33/1. 19. - (1954): Verwitterung und Bodenbildung auf basischen Eruptivgesteinen. Ibid. 34/2. 20. - (1958a): Las bases científicas de la Eadafología. Anales de Edaf. y Fis. Veg. $X V I I / 11$. 21. - (1958b): Welches sind nach den Erfahrungen der Bodenkunde die maßgebenden Klimafaktoren? Peterm. Geogr. Mitt. H. 2. 22. - (1959): Die Gesetze der Bodenbildung. N. Jb. f. Geol. Paläont. Mh. 9. 23. - (1960): Die Lößböden. Schweiz. min. petr. Mitt. 4o/2. 24. Scheffer, F. und Schachtschnaber., P. (1952): Bodenkunde. Stuttgart. 25. Schoenefeldt, O. (1936): Wasserhaushalt und Dürrejahre. D. Ern. d. Pfl. 32/7. 26. VAGeLER, P. (1930): Grundriß der tropischen und subtropischen Bodenkunde. Berlin. 27. von KneBEL, W. (1906): Höhlenkunde. Braunschweig.

\section{LE CYCLE DE L'EAU DANS L'ATMOSPHÈRE}

Si l'on connaît le cycle de l'eau dans l'atmosphère on n'a pourtant jamais réussi à le définir mathématiquement parce qu'on est incapable de mesurer exactement l'évaporation. Lang propose une définition mathématique de l'humidité en établissant le quotient des précipitations par la température moyenne. Il pense en effet qu'à chaque état d'humidité correspond un certain type de formation de sol, ce qui est du reste prouvé par les lois de chimie, de pétrographie et de géologie. Le facteur d'humidité de Lang indique les relations existant entre la température de l'air, la masse et la température des précipitations; il comprend ainsi les problèmes de répartition d'eau et de chaleur dans l'atmosphère. L'énergie absorbée annuellement par le sol détermine en fin de compte la formation d'un sol forestier endopercolatif et se trouve en équilibre avec le climat local et la végétation. 